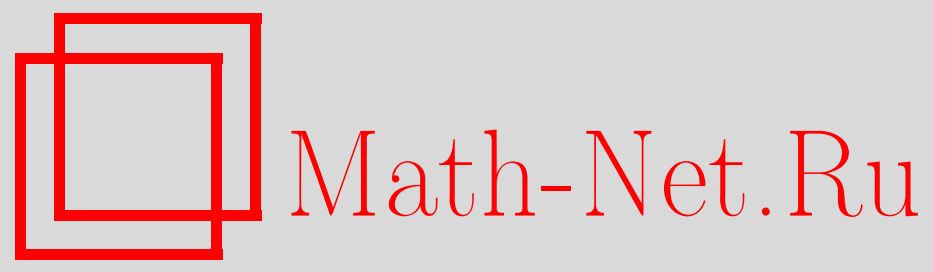

М. В. Козлов, О больших уклонениях ветвящихся процессов в случайной среде: геометрическое распределение числа потомков, Дискрет. матем., 2006, том 18, выпуск 2, 29 47

DOI: https://doi.org/10.4213/dm44

Использование Общероссийского математического портала Math-Net.Ru подразумевает, что вы прочитали и согласны с пользовательским соглашением http://www.mathnet.ru/rus/agreement

Параметры загрузки:

IP : 3.89 .185 .249

26 апреля 2023 г., 15:55:59 


\title{
О больших уклонениях ветвящихся процессов в случайной среде: геомтрическое распределение числа потомков
}

\author{
() 2006 г. М. В. Козлов
}

\begin{abstract}
Рассматривается ветвящийся процесс $Z_{\boldsymbol{n}}$ с геометрическим распределением числа непосредственных потомков в случайной среде, представляющей собой последовательность независимых одинаково распределенных случайных величин (модель СмитаВилкинсона). Найдена асимптотика вероятностей больших уклонений $\mathbf{P}\left(\ln Z_{n}>\theta n\right)$, $\theta>0$, в предположении, что шаг сопровождающего случайного блуждания $S_{n}$ удовлетворяет условию Крамера. Эта асимптотика следует за асимптотикой вероятностей больших уклонений $\mathbf{P}\left(S_{n}>\theta n\right)$ в случаях надкритического, критического, умеренно и промежуточно докритического процессов. В строго докритическом случае для $\theta$, больших некоторого $\theta^{*}$, сохраняется та же асимптотика (при $\theta \leqslant \theta^{*}$ вероятности больших уклонений имеют другую асимптотику).

Работа выполнена при поддержке Российского фонда фундаментальных исследований, проект 04-01-00700, и поддержке DFG, проект 436 RUS 113/722.
\end{abstract}

\section{1. Введение}

Рассмотрим марковскую цепь $Z_{n}, n=0,1,2, \ldots, Z_{0}=1$, с целыми неотрицательными состояниями и вероятностями перехода

$$
P_{i j}=\int P_{i j}(y) Q(d y), \quad i, j=0,1,2, \ldots,
$$

представляющими собой смесь вероятностей перехода $P_{i j}(y)$, соответствующих при каждом фиксированном $y \in$ У некоторому ветвящемуся процессу Гальтона-Ватсона с дробно-линейной производящей функщией. Именно,

$$
\begin{gathered}
f_{y}(s)=1-\left(\frac{1}{a(y)(1-s)}+\frac{b(y)}{2 a(y)^{2}}\right)^{-1}, \quad a(y), b(y) \geqslant 0, \\
\sum_{j=0}^{\infty} P_{i j}(y) s^{j}=f_{y}(s)^{i}, \quad i=0,1,2, \ldots, \quad|s| \leqslant 1 .
\end{gathered}
$$

Предполагается, что

$$
a(y)=f_{y}^{\prime}(1), \quad b(y)=f_{y}^{\prime \prime}(1)
$$


- измеримые функщии на некотором измеримом пространстве (У, $\mathscr{B}), Q$ - вероятностная мера на нем, $Q$-мера множества тех $y$, для которых $a(y)=0$, равна нулю.

Марковскую цепь $Z_{n}$, как и обычный ветвящийся процесс, полезно определить в терминах случайных величин. Введем две системы случайных элементов, последовательность $\eta .=\left(\eta_{0}=\eta, \eta_{1}, \eta_{2}, \ldots\right)$ независимых одинаково распределенных случайных элементов пространства (У, В) с распределением вероятностей $Q$, называемую случайной средой, и матрищу $\zeta_{i n}, i \geqslant 1, n=0,1,2, \ldots$, случайных величин построчно независимых, а в пределах строки условно независимых относительно случайной среды $\eta$. с общей (не зависящей от $i$ ) условной производящей функщией

$$
\mathbf{E}\left(s^{\zeta_{\text {in }}} \mid \eta .\right)=f_{\eta_{n}}(s) \text { п.н., } \quad|s| \leqslant 1 \text {. }
$$

Нетрудно видеть, что рекуррентное соотношение

$$
Z_{n+1}=\sum_{i=1}^{Z_{n}} \zeta_{i n}, \quad n=0,1,2, \ldots, \quad Z_{0}=1
$$

(где сумма по пустому множеству индексов полагается равной нулю) задает марковскую цепь с вероятностями перехода $(1)$. Процесс $\left(Z_{n}\right)$ называют ветвящимся процессом в случайной среде.

Благодаря тому, что класс дробно-линейных преобразований замкнут относительно суперпозищии, справедлива следующая формула (см. [1]):

$$
1-f_{y_{0}}\left(f_{y_{1}}\left(\ldots f_{y_{m}}(s) \ldots\right)\right)=\left(\left((1-s) \prod_{i=0}^{m} a\left(y_{i}\right)\right)^{-1}+\sum_{j=0}^{m-1} \frac{b\left(y_{j}\right)}{2 a\left(y_{j}\right)^{2}}\left(\prod_{i=0}^{j-1} a\left(Y_{i}\right)\right)^{-1}\right)^{-1} .
$$

Полагая

$$
\begin{aligned}
& X_{i}=\ln f_{\eta_{i-1}}^{\prime}(1), \quad S_{n}=\sum_{i=1}^{n} X_{i}, \quad i, n=1,2, \ldots, \quad S_{0}=0, \\
& U_{j}=e^{-S_{j}}, \quad V_{m}=\sum_{j=0}^{m-1} \frac{b\left(\eta_{j}\right)}{2 a\left(\eta_{j}\right)^{2}} U_{j}, \quad j \geqslant 0, \quad m \geqslant 1 \text {, }
\end{aligned}
$$

получаем, что

$$
\mathbf{E}\left(s^{Z_{n}} \mid \eta .\right)=1-\left\{(1-s)^{-1} U_{n}+V_{n}\right\}^{-1} \text { п.н. }
$$

Всюду далее предполагается, что случайная величина $X=\ln f_{\eta}^{\prime}(1)$ не вырождается в постоянную. Элементарные выкладки приводят к формуле

$$
\mathbf{P}\left(Z_{n}>k\right)=\mathbf{E}\left\{\left(V_{n}+U_{n}\right)^{-1}\left(1+V_{n}^{-1} U_{n}\right)^{-k}\right\}
$$

Случайное блуждание $\left(S_{n}\right)$ называется сопровождающим для ветвящегося процесса в случайной среде $\left(Z_{n}\right)$, оно входит явным образом в точные формулы (5), (6) для условной производящей функщии случайной величины $Z_{n}$ и для хвостов распределения вероятностей $Z_{n}$. Оно, однако, имеет принципиальное значение и в случае произвольных 
(а не только дробно-линейных) производящих функций $f_{y}(s)$. Заметим, что при $Z_{n} \gg 1$ справедливо приближенное равенство

$$
\frac{Z_{n+1}}{Z_{n}}=Z_{n}^{-1} \sum_{i=1}^{Z_{n}} \zeta_{i n} \cong f_{\eta_{n}}^{\prime}(1),
$$

соответствующее условному закону больших чисел. В результате

$$
\ln Z_{n+1} \cong \ln Z_{n}+X_{n+1}, \quad Z_{n} \gg 1,
$$

а приближенное равенство тем точнее, чем больше $Z_{n}$. Указанное соображение служит ключом к многим результатам теории ветвящихся процессов в случайной среде (см., например, [2-7] и приведенную там библиографию). В настоящей работе мы показываем, что при определенных условиях большие уклонения процесса $\ln Z_{n}$ в частном случае (20) следуют за большими уклонениями сопровождающего блуждания $S_{n}$, если шаг этого блуждания подчиняется условию Крамера.

\section{2. Сведения из теории суммирования независимых случайных величин}

Пусть $X, X_{1}, X_{2}, \ldots$ - последовательность независимых одинаково распределенных случайных величин с невырожденной функщией распределения $F(x)$,

$$
S_{n}=\sum_{i=1}^{n} X_{i}, \quad S_{0}=0 .
$$

Введем ряд обозначений, полагая, что соответствующие величины определены равенствами

$$
\begin{array}{rlrl}
R(h) & =\mathrm{E} e^{h X}=\int e^{h x} d F(x), & \mu & =\mathrm{E} X, \quad \sigma^{2}=\mathrm{D} X, \\
\rho & =\mathrm{E}(X-\mu)^{3} \sigma^{-3}, & \rho^{*}=\mathrm{E}|X-\mu|^{3} \sigma^{-3} .
\end{array}
$$

Будем говорить, что случайная величина $X$ и ее функция распределения $F_{X}$ удовлетворяют (двустороннему) условию Крамера, если $R(h)<\infty$ в некоторой окрестности точки $h=0$. Если $R(h)<\infty$ в правой полуокрестности точки $h=0$, то говорим, что выполнено правостороннее условие Крамера.

В предположении, что выполнено правостороннее условие Крамера и $\mu$ конечно, введем следующие обозначения:

$$
\begin{array}{rlrl}
h^{+} & =\sup \{h: R(h)<\infty\}, & m(h) & =R^{\prime}(h) / R(h), \\
\sigma^{2}(h) & =m^{\prime}(h)(>0), & \theta^{+} & =\lim _{h \rightarrow h^{+}} m(h), \\
h(\theta) & - \text { корень уравнения } m(h)=\theta & \text { при } \mu & <\theta<\theta^{+}, \\
\Lambda(\theta) & =h(\theta) \theta-\ln R(h(\theta)), & S_{n}^{h)} & =\sum_{i=1}^{n} X_{i}^{(h)}, \\
F^{(h)}(x) & =R(h)^{-1} \int_{-\infty}^{x} e^{h y} d F(y), & 0<h<h^{+},
\end{array}
$$


где $X^{(h)}, X_{1}^{(h)}, X_{2}^{(h)}, \ldots$ - независимые одинаково распределенные случайные величины с функцией распределения $F^{(h)}$.

Отметим, что $\mathbf{E} X^{(h)}=m(h), \mathbf{D} X^{(h)}=\sigma^{2}(h)$. В случае необходимости в обозначения (7), (8) будет вводиться нижний индекс, указывающий, о какой случайной величине идет речь: $R_{X}(h), m_{X}(h), h_{X}^{+}$и т. д.

Имеет место следующая асимптотика вероятностей больших уклонений для сумм $S_{n}$ [8]: если $n \rightarrow \infty$, то равномерно по $\theta$ из любого компакта, содержащегося в $\left(\mu, \theta^{+}\right)$,

$$
\mathbf{P}\left(S_{n}>\theta n\right) \sim(\sqrt{2 \pi n} h(\theta) \sigma(h(\theta)))^{-1} \exp \{-\Lambda(\theta) n\},
$$

если функция распределения $F$ нерешетчатая; в решетчатом случае в правую часть (9) следует добавить множитель

$$
d(1-\exp \{-d h(\theta)\})
$$

где $d-$ максимальный шаг решетки. Здесь и далее $a_{n} \sim b_{n}$ при $n \rightarrow \infty$ означает, что $a_{n} / b_{n} \rightarrow 1$ при $n \rightarrow \infty$. Отметим еще неравенства

$$
\begin{aligned}
\mathbf{P}\left(S_{n}>x\right) & =\mathbf{P}\left(e^{h S_{n}}>e^{h x}\right) \leqslant e^{-h x} R(h)^{n}, \quad h>0, \\
\mathbf{P}\left(S_{n}>\theta n\right) & \leqslant e^{-h(\theta) \theta n} R(h(\theta))^{n}=\exp \{-\Lambda(\theta) n\} .
\end{aligned}
$$

Имеет место следующее неравенство для уклонения распределения вероятностей случайной величины $S_{n}$ от нормальной функции распределения $\Phi(x)$ [9]:

$$
\left|\mathbf{P}\left(\frac{S_{n}-n \mu}{\sigma \sqrt{n}} \leqslant x\right)-\Phi(x)\right| \leqslant \frac{A \rho^{*}}{\sqrt{n}\left(1+|x|^{3}\right)},
$$

где $\mathscr{A}$ - универсальная постоянная. В частности, отбрасывая в (13) множитель, зависящий от $x$, получаем неравенство Берри-Эссеена.

Введем обозначение

$$
\Psi_{r}^{(n)}(x)=\Phi(x)+\frac{r}{6 \sqrt{n}}\left(x^{2}-1\right) \varphi(x), \quad \varphi(x)=\Phi^{\prime}(x), \quad r>0 .
$$

Если $\rho^{*}<\infty$, то в случае нерешетчатой функции распределения $F$ при $n \rightarrow \infty$ имеет место асимптотическое разложение

$$
\mathbf{P}\left(\frac{S_{n}-n \mu}{\sigma \sqrt{n}} \leqslant x\right)=\Psi_{\rho}^{(n)}(x)+o(1 / \sqrt{n}),
$$

равномерно по $x$, в решетчатом случае (15) справедливо в средних точках решетки с шагом $d_{n}=d /(\sigma \sqrt{n})$ (см. [10]). Положим при $r>0$

$$
\psi_{r}^{(n)}(x)=\frac{d \Psi_{r}^{(n)}(x)}{d x}=\frac{1}{\sqrt{2 \pi}} e^{-x^{2} / 2}\left(1+\frac{r}{6 \sqrt{\pi}}\left(x^{3}-3 x\right)\right)
$$

Заметим, что при всех $x, n$

$$
\left|\psi_{r}^{(n)}(x)\right| \leqslant \mathscr{A}_{r} e^{-x^{2} / 4}
$$




\section{4. Доказательство теоремы 1}

В предположении (20) вероятность (21) может быть представлена в виде (см. (6))

$$
\mathbf{E}\left\{V_{n+1}^{-1}\left(1+V_{n}^{-1} U_{n}\right)^{-k_{n}}\right\}, \quad U_{j}=e^{-S_{j}}, \quad V_{j}=\sum_{i=0}^{j-1} U_{i},
$$

где $k_{n}$ обозначает целую часть числа $e^{\theta n}$. Возьмем $\varkappa$ из условия $\theta / 2<\varkappa<\theta$ и представим (24) в виде суммы

$$
E_{1}^{(n)}+E_{2}^{(n)}, \quad E_{1}^{(n)}=\mathrm{E}\left\{\ldots ; S_{n} \geqslant \varkappa n\right\}, \quad E_{2}^{(n)}=\mathbf{E}\left\{\ldots ; S_{n}<\varkappa n\right\},
$$

где многоточие заменяет выражение, стоящее под знаком математического ожидания в (24). Слагаемое $E_{2}^{(n)}$ оценивается достаточно просто и при $n \rightarrow \infty$ оказывается величиной бесконечно малой по сравнению с $E_{1}^{(n)}$ (см. лемму 12). Вывод асимптотики слагаемого $E_{1}^{(n)}$ содержится в леммах 1-11.

Представим $E_{1}^{(n)}$ в интегральной форме:

$$
\int_{s_{n} \geqslant x n} \ldots \int\left(\sum_{j=0}^{n} e^{-s_{j}}\right)^{-1}\left(1+\left(\sum_{j=0}^{n-1} e^{-s_{j}}\right)^{-1} e^{-s_{n}}\right)^{-k_{n}} \prod_{i=1}^{n} d F\left(x_{i}\right),
$$

где $s_{j}=x_{1}+\ldots+x_{j}$. Фиксируем $\theta$ и введем упрощенный вариант обозначений (7), (8), полагая

$$
\begin{aligned}
& \bar{h}=h(\theta), \quad \bar{\sigma}^{2}=\sigma(\bar{h})^{2}, \quad \bar{F}=F^{(\bar{h})}, \quad \bar{X}=X^{(\bar{h})}, \quad \bar{\rho}=\rho_{\bar{X}}, \\
& \bar{\rho}^{*}=\rho_{\bar{X}}^{*}, \quad \bar{S}_{j}=S_{j}^{(\bar{h})}, \quad \bar{V}_{n}=\sum_{j \leqslant n-1} e^{-\bar{S}_{j}}, \quad \bar{V}_{\infty}=\bar{V} .
\end{aligned}
$$

Проведем в интеграле (26) замену меры по формуле (преобразование Крамера)

$$
\prod_{i=1}^{n} d F\left(x_{i}\right)=R(\bar{h})^{n} e^{-\bar{h} s_{n}} \prod_{i=1}^{n} d \bar{F}\left(x_{i}\right) .
$$

Вынесем за знак интеграла множитель $R(\bar{h})^{n} e^{-\bar{h} \theta n}$, так что остающийся интеграл принимает вид

$$
\begin{aligned}
\int_{s_{n} \geqslant x n} \ldots \int_{j=0} e^{-\bar{h}\left(\bar{s}_{n}-\theta n\right)}\left(\sum_{j=0}^{n} e^{-s_{j}}\right)^{-1} & \left(1+\left(\sum_{j=0}^{n-1} e^{-s_{j}}\right)^{-1} e^{-s_{n}}\right)^{-k_{n}} \prod_{i=1}^{n} d \bar{F}\left(x_{i}\right) \\
& =\mathbf{E}\left\{e^{-\bar{h}\left(\bar{S}_{n}-\theta n\right)} \bar{V}_{n+1}^{-1}\left(1+\bar{V}_{n}^{-1} e^{-\bar{S}_{n}}\right)^{-k_{n}} ; \bar{S}_{n} \geqslant x n\right\} .
\end{aligned}
$$

Заметив, что $\mathbf{E} \bar{X}_{i}=\theta$, положим

$$
\begin{aligned}
\hat{X}_{i} & =\bar{X}_{i}-\theta, \quad \hat{S}_{n}=\bar{S}_{n}-\theta n, \\
g_{n}(u, v) & =\left(v+e^{-\theta n-u}\right)^{-1}\left(1+v^{-1} e^{-\theta n-u}\right)^{-k_{n}}, \quad v \geqslant 1, \\
I_{n}(A \times B) & =\int_{A} \int_{B} e^{-\bar{h} u} g_{n}(u, v) \mathbf{P}\left(\hat{S}_{n} \in d u, \bar{V}_{n} \in d v\right)
\end{aligned}
$$


где постоянная $\mathscr{A}_{r}$ зависит от $r$. Из (15)-(17) легко следует, что при любом $\Delta>0$ и всех $a, b$ таких, что $b-a \geqslant \Delta$, выполнено неравенство

$$
\mathbf{P}\left(S_{n} \in(a, b]\right) \leqslant \mathscr{A}^{\prime}(b-a) /(\sigma \sqrt{n}),
$$

где постоянная $\mathscr{A}^{\prime}$ зависит от $F$ и $\Delta$; и равномерно по $a \leqslant b$ таким, что $a, b=o(\sqrt{n}$ ) (то есть для $|a|,|b| \leqslant \varepsilon_{n} \sqrt{n}$ и любой последовательности $\varepsilon_{n}>0$, стремящейся к нулю при $n \rightarrow \infty)$ справедливо соотношение

$$
\mathbf{P}\left(S_{n}-n \mu \in(a, b]\right)=(b-a) /(\sigma \sqrt{2 \pi n})+o(1 / \sqrt{n})
$$

при $n \rightarrow \infty$.

\section{3. Основные результаты}

Теорема 1. Предположим, что условное распределение вероятностей числа непосредственных потомков частицы при условии среды является геометрическим (см. (2)),

$$
\frac{b(y)}{2 a(y)^{2}}=1, \quad y \in \mathcal{Y},
$$

случайная величина $X=\ln f_{\eta}^{\prime}(1)$ удовлетворяет правостороннему условию Крамера $и$ $0 \leqslant \mu=\mathbf{E} X<\infty$. Тогда при $n \rightarrow \infty$ равномерно по $\theta$ из любого компакта, содержсачегося в $\left(\mu, \theta^{+}\right)$, имеет место асимптотическое соотночение

$$
\mathbf{P}\left(\ln Z_{n}>\theta n\right) \sim I(\theta) \mathbf{P}\left(S_{n}>\theta n\right),
$$

гдe

$$
\begin{aligned}
I(\theta) & =\Gamma(h(\theta)) \int_{1}^{\infty} v^{h(\theta)-1} d G_{\theta}(v), \\
G_{\theta}(v) & =\mathbf{P}\left(\sum_{j=0}^{\infty} \exp \left\{-S_{j}^{(h(\theta))}\right\} \leqslant v\right)
\end{aligned}
$$

и Г(·) - гамма-функция.

Заметим, что для вероятностей $\mathbf{P}\left(S_{n}>\theta n\right)$ справедлива асимптотика (9).

Теорема 2. Предположим, что выполнены все условия теоремы 1 , кроме условия $\mu \geqslant 0$, которое заменим на $\mu<0$. Предположим, что

$$
h(0)<h^{+}, \quad m(h(0))=0=R^{\prime}(h(0)) .
$$

Тогда асимптотика (21) имеет место равномерно по $\theta$ из любого компакта, содержащегося в $\left(0, \theta^{+}\right)$, если $h(0) \leqslant 1$, и содержашегося в $\left(\theta^{*}, \theta^{+}\right)$, если $h(0)>1$, и найдется такое (единственное) $h^{*} \in\left(1, h^{+}\right)$, что $R\left(h^{*}\right)=R(1)$, при этом $\theta^{*}$ определяется уравнением $m\left(\theta^{*}\right)=h^{*}$. 
при $A \subseteq(-\infty, \infty), B \subseteq[1, \infty)$. В этих обозначениях математическое ожидание (28) есть $I_{n}([-(\theta-\chi) n, \infty) \times[1, \infty))$. Далее мы рассматриваем только нерешетчатый случай, для решетчатой функщии распределения $F$ видоизменения в рассуждениях не представляют труда. Мы покажем, что при $n \rightarrow \infty$

$$
I_{n}([-(\theta-\varkappa) n, \infty) \times[1, \infty)) \sim I(\theta)(\sqrt{2 \pi n} \bar{h} \bar{\sigma})^{-1},
$$

откуда следует (см. (8), (9), что при $n \rightarrow \infty$

$$
E_{1}^{(n)}=R(\bar{h})^{n} e^{-\bar{h} \theta n} I_{n}([-(\theta-\varkappa) n, \infty) \times[1, \infty)) \sim I(\theta) \mathbf{P}\left(S_{n}>\theta n\right) .
$$

Перейдем к выводу основного предельного соотношения (32). Отметим, что некоторые из нижеследующих лемм устанавливаются при более широких предположениях, чем это требуется для доказательства (32).

Лемма 1 ([11]). Пусть $\xi, \xi_{1}, \xi_{2}, \ldots$ - последовательность (невырождаючихся в постоянную) независимых одинаково распределенных случайных величин, удовлетворяющих правостороннему условию Крамера. Пусть

$$
\mu_{\xi}=\mathbf{E} \xi<0, \quad q=\sup \left\{h: R_{\xi}(h) \leqslant 1\right\}, \quad \eta_{n}=\sum_{i=1}^{n} \xi_{i}, \quad \eta_{0}=0, \quad \bar{\eta}_{n}=\max _{0 \leqslant k \leqslant n} \eta_{k} .
$$

Тогда

$$
\mathbf{P}\left(\bar{\eta}_{n} \geqslant y\right) \leqslant e^{-q y}, \quad y \geqslant 0 .
$$

Доказательство. Введем несобственную случайную величину $\tau_{y}$ - момент первого попадания случайного блуждания $\eta_{n}$ в $[y, \infty)$. При $h>0$

$$
\begin{aligned}
R_{\xi}(h)^{n} & =\mathbf{E} e^{h \eta_{n}} \geqslant \sum_{k=1}^{n} \mathbf{E}\left\{e^{h \eta_{k}} ; \tau_{y}=k\right\} \mathbf{E} e^{h\left(\eta_{n}-\eta_{k}\right)} \\
& \geqslant R_{\xi}(h)^{n} e^{h y} \sum_{k=1}^{n} \mathbf{P}\left(\tau_{y}=k\right) R_{\xi}(h)^{-k} .
\end{aligned}
$$

Функция $R_{\xi}(h)$ строго выпукла, $R_{\xi}^{\prime}(0)=\mu_{\xi}<0, R_{\xi}(0)=1$, так что $R_{\xi}(h)<1$ в некоторой правой полуокрестности точки $h=0$. Поэтому указанное в условии леммы число $q$ положительно, а при $0<h<q$

$$
\mathbf{P}\left(\bar{\eta}_{n} \geqslant y\right)=\mathbf{P}\left(\tau_{y} \leqslant n\right) \leqslant \sum_{k=1}^{n} \mathbf{P}\left(\tau_{y}=k\right) R_{\xi}(h)^{-k} \leqslant e^{-h y} .
$$

Остается перейти в этом неравенстве к пределу при $h \rightarrow q$.

Лемма 2. Для любого $0 \leqslant \alpha<\theta$ существует такое $q_{\alpha}>0$, что

$$
\mathbf{P}\left(\min _{k \geqslant 0}\left(\bar{S}_{k}-\alpha k\right) \leqslant-y\right) \leqslant e^{-q_{\alpha} y}, \quad y>0,
$$

причем $q_{\alpha}=\bar{h}$ при $\alpha \leqslant \bar{\alpha}=\bar{h}^{-1} \ln R_{X}(\bar{h})$. 
Доказательство. Заметим, что $\bar{h} \theta-\ln R_{X}(\bar{h})^{-1}=\Lambda(\theta)>0$, а потому $\bar{h} \theta-\bar{h} \bar{\alpha}>0$ или $\bar{\alpha}<\theta$. Неравенство (35) получается подстановкой $\xi_{i}=-\bar{X}_{i}+\alpha$ в неравенство (34), если учесть, что

$$
\begin{gathered}
\mathrm{E}\left(-\bar{X}_{i}+\alpha\right)=-\theta+\alpha<0, \quad \mathbf{E} e^{h(-\bar{X}+\alpha)}=e^{h \alpha} \mathrm{E} e^{-h \bar{X}}, \\
\mathbf{E} e^{-h \bar{X}}=R_{X}(\bar{h})^{-1} \int e^{(\bar{h}-h) x} d F_{X}(x)=R_{X}(\bar{h})^{-1} R_{X}(\bar{h}-h)<\infty
\end{gathered}
$$

при $\bar{h}-h_{X}^{+} \leqslant h \leqslant \bar{h}$. При этом

$$
q_{\alpha}=\sup \left\{h e^{h \alpha} R_{X}(\bar{h})^{-1} R_{X}(\bar{h}-h) \leqslant 1\right\} .
$$

Функция $R_{X}(h)$ строго выпукла, $R_{X}^{\prime}(0)=\mu \geqslant 0, R_{X}(0)=1$, так что на интервале $\left(0, h_{X}^{+}\right)$ она возрастает и потому больше 1 . Полагая $h=\bar{h}$ в неравенстве под знаком супремума в (36), получаем, что либо неравенство $e^{\bar{h} \alpha} R_{X}(\bar{h})^{-1} \leqslant 1$, либо неравенство $\bar{h} \alpha-\ln R_{X}(\bar{h}) \leqslant 0$ выполняется при $\alpha \leqslant \bar{\alpha}$.

Лемма 3. При любых $0 \leqslant \alpha<\beta<\theta, \Delta>0$, всех $a, b$ таких, что $b-\Delta \geqslant a \geqslant \beta n$, выполняется неравенство

$$
\mathbf{P}\left(\bar{S}_{n} \in(a, b], \min _{0 \leqslant k \leqslant n}\left(\bar{S}_{k}-\alpha k\right) \leqslant-y\right) \leqslant(C(b-a) / \sqrt{n}) e^{-q_{\alpha} y}, \quad y>0,
$$

где $q_{\alpha}$ определено в (36); постоянная $C$ зависит от $\alpha, \beta, \Delta, \bar{\sigma}, \bar{\rho}^{*}$.

Доказательство. Введем несобственную случайную величину $\tau_{y}-$ момент первого попадания блуждания $-\bar{S}_{k}+\alpha k$ в $[y, \infty), y>0$, и представим вероятность (37) в виде

$$
\sum_{k=1}^{n} \mathbf{P}\left(\bar{S}_{n} \in(a, b], \tau_{y}=k\right)=\sum_{k=1}^{n} \mathbf{E}\left\{I_{\left\{\tau_{y}=k\right\}} \mathbf{E}\left(I_{\left\{\bar{S}_{n} \in(a, b]\right\}} \mid \bar{X}_{i}, i \leqslant k\right)\right\},
$$

где $I_{\{\}\}}$означает индикатор события $\{\cdot\}$. При заданном $1 \leqslant k \leqslant n$ фиксируем значения случайной величины $\bar{X}_{i}, i \leqslant k$, отвечающие событию $\tau_{y}=k$, и пусть при этом $\bar{S}_{k}=\alpha k-z$, $z \geqslant y$. Будем считать, что условное математическое ожидание в (38) принимает при $\bar{S}_{k}=\alpha k-z$ значение

$$
\mathbf{P}\left(\bar{S}_{n-k}+\alpha k-z \in(a, b]\right)=\mathbf{P}\left(\hat{S}_{n-k}+\theta(n-k)+\alpha k-z \in(a, b]\right) .
$$

Положим для краткости

$$
a_{k}^{\prime}=a-\theta(n-k)-\alpha k+z, \quad b_{k}^{\prime}=b-\theta(n-k)-\alpha k+z, \quad \delta=(\beta-\alpha) /(2 \theta) .
$$

Для оценивания вероятностей (39) при $k \leqslant n(1-\delta)$ воспользуемся неравенством (18) и получим оценку

$$
\mathbf{P}\left(\bar{S}_{n-k}+\alpha k-z \in(a, b]\right) \leqslant \mathscr{A}^{\prime} \delta^{-1 / 2}(b-a) /(\bar{\sigma} \sqrt{n})
$$

При $n-1 \geqslant k>n(1-\delta)$ для оценивания вероятностей (39) воспользуемся неравенством (13). Оценку сверху запишем в виде суммы двух слагаемых

$$
\begin{gathered}
\left(\Phi\left(\frac{b_{k}^{\prime}}{\bar{\sigma} \sqrt{n-k}}\right)-\Phi\left(\frac{a_{k}^{\prime}}{\bar{\sigma} \sqrt{n-k}}\right)\right), \\
\frac{A \bar{\rho}^{*}}{\sqrt{n-k}}\left(\left(\frac{a_{k}^{\prime}}{\bar{\sigma} \sqrt{n-k}}\right)^{-3}+\left(\frac{b_{k}^{\prime}}{\bar{\sigma} \sqrt{n-k}}\right)^{-3}\right) \leqslant \frac{A \bar{\rho}^{*}}{\sqrt{n-k}}\left(\frac{\left(a_{k}^{\prime}\right)^{-1}+\left(b_{k}^{\prime}\right)^{-1}}{\bar{\sigma} \sqrt{n-k}}\right)^{3},
\end{gathered}
$$


здесь учтено, что поскольку $z>0, a \geqslant \beta n, k>n(1-\delta)$, то

$$
b_{k}^{\prime}-\Delta \geqslant a_{k}^{\prime}>\beta_{n}-\theta(n-n(1-\delta))-\alpha n=(\beta-\alpha-\theta \delta) n=1 / 2(\beta-\alpha) n>0 .
$$

Используя эти неравенства в обоих слагаемых в (41), получаем для них, соответственно, оценки сверху

$$
\begin{gathered}
\varphi\left(\frac{(\beta-\alpha) n}{2}\right) \frac{b_{k}^{\prime}-a_{k}^{\prime}}{\bar{\sigma} \sqrt{n-k}}=\frac{b-a}{\bar{\sigma} \sqrt{2 \pi(n-k)}} \exp \left\{-\frac{((\beta-\alpha) n)^{2}}{8}\right\}, \\
\frac{A \bar{\rho}^{*}}{\sqrt{(n-k)}}\left(\frac{(\beta-\alpha) n}{\bar{\sigma} \sqrt{(n-k)}}\right)^{-3} \leqslant \frac{A \bar{\rho}^{*} \bar{\sigma}^{3}}{(\beta-\alpha)^{3}} n^{-2} \leqslant \frac{A \bar{\rho}^{*} \bar{\sigma}^{3}}{(\beta-\alpha)^{3} \Delta} \frac{b-a}{\sqrt{n}} .
\end{gathered}
$$

Эти оценки вместе с (40) приводят к неравенству

$$
I_{\left\{\tau_{y}=k\right\}} \mathbf{E}\left(I_{\left\{\bar{S}_{n} \in(a, b]\right\}} \mid \bar{X}_{i}, i \leqslant k\right) \leqslant I_{\left\{\tau_{y}=k\right\}} C(b-a) / \sqrt{n} \text { п.н. }
$$

(отметим, что событие $\left\{\bar{S}_{n} \in(a, b], \tau_{y}=n\right\}$ имеет нулевую вероятность). Подставляя (42) в (38), получаем для вероятности (37) оценку сверху

$$
(C(b-a) / \sqrt{n}) \sum_{k=1}^{n} \mathbf{P}\left(\tau_{y}=k\right)=(C(b-a) / \sqrt{n}) \mathbf{P}\left(\min _{0 \leqslant k \leqslant n}\left(\bar{S}_{k}-\alpha k\right)<-y\right) .
$$

Остается воспользоваться оценкой (35).

Лемма 4. В предположениях и обозначениях леммы 3 и дополнительном ограничении $\alpha>0$ имеют место неравенства

$$
\begin{aligned}
\mathbf{P}\left(\bar{S}_{n} \in(a, b], \bar{V}_{n}>\left(1-e^{-\alpha}\right)^{-1} e^{y}\right) \leqslant(C(b-a) / \sqrt{n}) e^{-q_{\alpha} y}, & & y>0, \\
\mathbf{P}\left(\bar{V}_{n}>\left(1-e^{-\alpha}\right)^{-1} e^{y}\right) \leqslant e^{-q_{\alpha} y}, & & y>0,
\end{aligned}
$$

где $q_{\alpha}=\bar{h}$ nрu $0<\alpha \leqslant \bar{\alpha}$.

Доказательство. Если

$$
\min _{k \geqslant 0}\left(\bar{S}_{k}-\alpha k\right) \geqslant-y,
$$

To

$$
\bar{V}_{n} \leqslant \bar{V}=\sum_{k=0}^{\infty} e^{-\bar{S}_{k}+\alpha k} e^{-\alpha k} \leqslant e^{y} \sum_{k=0}^{\infty} e^{-\alpha k}=\left(1-e^{-\alpha}\right)^{-1} e^{y} .
$$

Отсюда следует, что

$$
\left\{\bar{V}_{n}>\left(1-e^{-\alpha}\right)^{-1} e^{y}\right\} \subseteq\left\{\min _{k \geqslant 0}\left(\bar{S}_{k}-\alpha k\right)<-y\right\} .
$$

Переходя здесь к вероятностям и применяя (35), получаем второе из неравенств (43). Первое получается применением неравенства (37). Лемма доказана.

Для последующего применения перепишем неравенства (43) при $\alpha=\bar{\alpha}$ в виде

$$
\mathbf{P}\left(\bar{S}_{n} \in(a, b], \bar{V}>v\right) \leqslant(C(b-a) / \sqrt{n}) \bar{C} v^{-\bar{h}}, \quad \mathbf{P}\left(\bar{V}_{n}>v\right) \leqslant \bar{C} v^{-\bar{h}}, \quad v \geqslant 1,
$$

где

$$
\bar{C}=\left(1-R_{X}(\bar{h})^{-\bar{h}^{-1}}\right)^{-\bar{h}}
$$


Лемма 5. Для любого $\Delta>0$ и всех $a, b$ таких, что $b-\Delta \geqslant a$, асимптотическое соотномение при $n \rightarrow \infty$

$$
\mathbf{P}\left(\bar{S}_{n} \in(a, b], \bar{V}_{n} \leqslant y\right)=((b-a) /(\bar{\sigma} \sqrt{2 \pi n}))(\mathbf{P}(\bar{V} \leqslant y)+o(1))
$$

выполняется во всех точках непрерывности функчии распределения случайной величины $\bar{V}$ равномерно по $a, b$, лежащим в окрестности точки $\theta n$ ширины о $(\sqrt{n})$.

Доказательство. Далее мы предполагаем, что $\mathbf{P}(\bar{V} \leqslant y)>0$, так как при $\mathbf{P}(\bar{V} \leqslant y)=0$ соотношение (45) сводится к (19). Положим

$$
\hat{a}_{n}=a-\theta n, \quad \hat{b}_{n}=b-\theta n \quad\left(\hat{b}_{n}-\Delta \geqslant \hat{a}_{n}\right),
$$

так что

$$
\left.\left\{\bar{S}_{n} \in(a, b]\right\}=\left\{\hat{S}_{n} \in \hat{a}_{n}, \hat{b}_{n}\right]\right\}
$$

Фиксируем произвольное значение $m<n$ и с учетом неравенства $\bar{V}_{m} \leqslant \bar{V}_{n}$ оценим вероятность в левой части (45) сверху вероятностью

$$
\begin{aligned}
\mathbf{P}\left(\hat{S}_{n} \in\left(\hat{a}_{n}, \hat{b}_{n}\right], \bar{V}_{m} \leqslant y\right) & =\int_{-\infty}^{\infty} \mathbf{P}\left(\hat{S}_{n-m}+s \in\left(\hat{a}_{n}, \hat{b}_{n}\right]\right) \mu_{m}(d s,[1, y]), \\
\mu_{m}(d s, d v) & =\mathbf{P}\left(\hat{S}_{m} \in d s, \bar{V}_{m} \in d v\right) .
\end{aligned}
$$

Интеграл (46) представим в виде суммы $I_{1}^{(n)}+I_{2}^{(n)}$, проводя интегрирование в (46) по областям $|s| \leqslant \sqrt[4]{n}$ и $|s|>\sqrt[4]{n}$ соответственно. Вероятность под интегралом $I_{1}^{(n)}$ заменим на асимптотическое разложение (15) и получим для нее выражение

$$
\begin{aligned}
& \Psi_{\bar{\rho}}^{(n-m)}\left(\frac{\hat{a}_{n}-s}{\bar{\sigma} \sqrt{n-m}}\right)-\Psi_{\bar{\rho}}^{(n-m)}\left(\frac{\hat{b}_{n}-s}{\bar{\sigma} \sqrt{n-m}}\right)+o\left(\frac{1}{\sqrt{n-m}}\right) \\
&=\psi_{\bar{\rho}}^{(n-m)}\left(\frac{\hat{c}_{n}-s}{\bar{\sigma} \sqrt{n-m}}\right) \frac{b-a}{\bar{\sigma} \sqrt{n-m}}+o\left(\frac{1}{\sqrt{n-m}}\right), \quad \hat{c}_{n} \in\left(\hat{a}_{n}, \hat{b}_{n}\right] .
\end{aligned}
$$

Так как $\hat{c}_{n}=o(\sqrt{n}),|s| \leqslant \sqrt[4]{n}$, при $n \rightarrow \infty$

$$
\begin{gathered}
\psi_{\bar{\rho}}^{(n-m)}\left(\left(\hat{c}_{n}-s\right) /(\bar{\sigma} \sqrt{n-m})\right)-1 / \sqrt{2 \pi}=o(1), \\
I_{1}^{(n)}=(((b-a) /(\bar{\sigma} \sqrt{2 \pi n}))(1+o(1))+o(1 / \sqrt{n})) \mu_{m}([-\sqrt[4]{n}, \sqrt[4]{n}],[1, y]),
\end{gathered}
$$

и

$$
\mu_{m}([-\sqrt[4]{n}, \sqrt[4]{n}],[1, y]) \leqslant \mathbf{P}\left(\bar{V}_{m} \leqslant y\right)
$$

где члены $o(1), o(1 / \sqrt{n})$ малы равномерно по $a, b, y$.

Учитывая, что случайная величина $\bar{X}$ удовлетворяет двустороннему условию Крамера (см. лемму 2) и применяя неравенство (11), получаем, что

$$
\mathbf{P}\left(\hat{S}_{m}>\sqrt[4]{n}\right) \leqslant R_{\hat{X}}(h)^{m} e^{-h \sqrt[4]{n}}
$$

при некотором $h>0$. Аналогичное неравенство имеет место для $\mathbf{P}\left(\hat{S}_{m}<-\sqrt[4]{n}\right)$. Поэтому

$$
I_{2}^{(n)} \leqslant \int_{|s|>\sqrt[4]{n}} \mu_{m}(d s,[1, y]) \leqslant \mathbf{P}\left(\left|\hat{S}_{m}\right|>\sqrt[4]{n}\right)=O(1) e^{-h \sqrt[4]{n}}
$$


Вместе с (49), (50) отсюда получаем оценку

$$
\varlimsup_{n \rightarrow \infty} \sqrt{n}\left(I_{1}^{(n)}+I_{2}^{(n)}\right) \leqslant((b-a) /(\bar{\sigma} \sqrt{2 \pi})) \mathbf{P}\left(\bar{V}_{m} \leqslant y\right) .
$$

Так как последовательность $\bar{V}_{m}$, возрастая, сходится к $\bar{V}$ п. н., для любого $\delta>0$ и всех достаточно больших $m$

$$
\mathbf{P}\left(\bar{V}-\delta \leqslant \bar{V}_{m} \leqslant \bar{V}\right) \geqslant 1-\delta .
$$

Отсюда следует, что

$$
\mathbf{P}\left(\bar{V}_{m} \leqslant y\right) \leqslant \mathbf{P}(\bar{V} \leqslant y+\delta)+\delta .
$$

Используя это неравенство в (51), получаем, что

$$
\varlimsup_{n \rightarrow \infty} \sqrt{n} \mathbf{P}\left(\bar{S}_{n} \in(a, b], \bar{V}_{n} \leqslant y\right) \leqslant(b-a) /(\bar{\sigma} \sqrt{2 \pi})(\mathbf{P}(\bar{V} \leqslant y+\delta)+\delta)
$$

равномерно по $a, b, \delta$.

Для получения нижней границы вероятности в левой части (45) используем разложение

$$
\bar{V}_{n}=\bar{V}_{m}+e^{-\bar{S}_{m}}\left(1+\sum_{j=m+1}^{n-1} e^{-\left(\bar{S}_{j}-\bar{S}_{m}\right)}\right)
$$

и заметим, что пара случайных величин $\bar{S}_{n}-\bar{S}_{m}=\hat{S}_{n}-\hat{S}_{m}, 1+\sum_{j=m+1}^{n-1} e^{-\left(\bar{S}_{j}-\bar{S}_{m}\right)}$ не зависит от пары $\hat{S}_{m}, \bar{V}_{m}$, а их совместное распределение вероятностей такое же, как у пары $\hat{S}_{n-m}, \bar{V}_{n-m}$. В результате вероятность в левой части (45) представляется в виде

$$
\int_{-\infty}^{\infty} \int_{1}^{\infty} \mathbf{P}\left(\hat{S}_{n-m}+s \in\left(\hat{a}_{n}, \hat{b}_{n}\right], v+e^{-s-\theta m} \bar{V}_{n-m} \leqslant y\right) \mu_{m}(d s, d v) .
$$

Оценим интеграл (55) снизу, сузив область интегрирования до множества

$$
D_{m, \delta}=\{|s| \leqslant \theta m / 2,1 \leqslant v \leqslant y-\delta\},
$$

где $\delta, 0<\delta<1$, будет в последующем выбираться сколь угодно малым. Ясно, что при $(s, v) \in D_{m, \delta}$

$$
\left\{\bar{V}_{n-m} \leqslant(y-v) e^{s+\theta m}\right\} \supseteq\left\{\bar{V}_{n-m} \leqslant \delta e^{\theta m / 2}\right\} .
$$

Таким образом, при $(s, v) \in D_{m, \delta}$ вероятность под интегралом (55) не меньше, чем

$$
\begin{aligned}
\mathbf{P}\left(\hat{S}_{n-m}+s \in\right. & \left.\left(\hat{a}_{n}, \hat{b}_{n}\right], \bar{V}_{n-m} \leqslant \delta e^{\theta m / 2}\right) \\
& =\mathbf{P}\left(\hat{S}_{n-m}+s \in\left(\hat{a}_{n}, \hat{b}_{n}\right]\right)-\mathbf{P}\left(\hat{S}_{n-m}+s \in\left(\hat{a}_{n}, \hat{b}_{n}\right], \bar{V}_{n-m}>\delta e^{\theta m / 2}\right) .
\end{aligned}
$$

Подставим (57) в интеграл (55), взятый по области (56), и заменим интеграл от разности вероятностей (57) на разность интегралов, обозначив их через $I_{+}^{(n)}$ и $I_{-}^{(n)}$.

К первой вероятности в правой части (57) применим асимптотическое разложение (47). Учитывая (48) и проводя интегрирование, получаем, что

$$
I_{+}^{n}=\left(\frac{b-a}{\bar{\sigma} \sqrt{2 \pi n}}(1+o(1))+o\left(\frac{1}{\sqrt{n}}\right)\right) \mathbf{P}\left(\left|\hat{S}_{m}\right| \leqslant \theta m / 2, \bar{V}_{m} \leqslant y-\delta\right) .
$$


Выберем $m$ зависящим от $\delta$ из условия

$$
\mathbf{P}\left(\left|\hat{S}_{m}\right|>\theta m / 2\right)<\delta .
$$

Отсюда и из неравенства $\bar{V}_{m} \leqslant \bar{V}$ получаем для вероятности в (58) границу снизу в виде

$$
\mathbf{P}(\bar{V} \leqslant y-\delta)-\delta \text {. }
$$

Вторую вероятность в (57) оценим сверху с использованием первого из неравенств (44) и, принимая во внимание (56), получаем для нее оценку сверху в виде

$$
(C(b-a) / \sqrt{n-m}) \bar{C} \delta^{-\bar{h}} e^{-\bar{h} \theta m / 2} .
$$

В результате интеграл $I_{-}^{(n)}$ оценивается сверху произведением выражения (60) на интеграл

$$
\int_{D_{m, \delta}} \int \mu_{m}(d s, d v)=\mathbf{P}\left(\left|\hat{S}_{m}\right| \leqslant \theta m / 2, \bar{V}_{m} \leqslant y-\delta\right) \leqslant \mathbf{P}(\bar{V} \leqslant y)+\delta
$$

(для $m$ таких, что выполнено (52)). Собирая вместе оценки (58)-(61), получаем для вероятности в левой части (45) оценку снизу в виде

$$
\begin{aligned}
(((b-a) /(\bar{\sigma} \sqrt{2 \pi n}))(1+o(1)) & +o(1 /(\sqrt{n})))(\mathbf{P}(\bar{V} \leqslant y-\delta)-\delta) \\
& -(C(b-a) / \sqrt{n-m}) \bar{C} \delta^{-\bar{h}} e^{-\bar{h} \theta m / 2}(\mathbf{P}(\bar{V} \leqslant y)+\delta) .
\end{aligned}
$$

Стандартные рассуждения приводят от оценок (62), (53) к соотношению (45). Именно, пусть $y$ - точка непрерывности функции распределения случайной величины $\bar{V}$. По любому $\varepsilon>0$ выберем $\delta>0$ так, что выполняются неравенства

$$
\mathbf{P}(\bar{V} \leqslant y+\delta)-\varepsilon \leqslant \mathbf{P}(\bar{V} \leqslant y) \leqslant \mathbf{P}(\bar{V} \leqslant y-\delta)+\varepsilon .
$$

Если $\delta$ уменьшить, то неравенства (63) сохраняются, пусть $\delta \leqslant \varepsilon$. По заданному $\delta$ выберем $m$ так, чтобы выполнялись неравенства (52) и неравенство

$$
C \bar{C} \delta^{-\bar{h}} e^{-\bar{h} \theta m / 2}<\varepsilon /(\sigma \sqrt{2 \pi}) .
$$

Будем выбирать $n$ настолько большим, чтобы остаточный член в асимптотическом разложении (47) был меньше, чем $\varepsilon / \sqrt{n}$. В таком случае, используя неравенства (63), (64) для оценки правых частей неравенств (53), (62), получаем при достаточно больших $n$ для вероятности в левой части (45) оценки сверху и снизу, соответственно,

$$
\begin{gathered}
((b-a) /(\bar{\sigma} \sqrt{2 \pi n}))\left((1+o(1))(\mathbf{P}(\bar{V} \leqslant y)+2 \varepsilon)+\varepsilon \Delta^{-1} \bar{\sigma} \sqrt{2 \pi}\right), \\
\frac{(b-a)}{(\bar{\sigma} \sqrt{2 \pi n})}\left(\left(1+o(1)-\frac{\varepsilon \bar{\sigma} \sqrt{2 \pi}}{\Delta}\right)(\mathbf{P}(\bar{V} \leqslant y)-2 \varepsilon)-\frac{\varepsilon(\mathbf{P}(\bar{V} \leqslant y)+\varepsilon)}{\sqrt{1-m / n}}\right) .
\end{gathered}
$$

Из (65), (66) вытекает, что верхний предел при $n \rightarrow \infty$ выражения

$$
\mathbf{P}\left(\hat{S}_{n} \in(a, b], \bar{V}_{n} \leqslant y\right)((b-a) /(\bar{\sigma} \sqrt{2 \pi n}))^{-1}
$$

не превосходит

$$
\mathbf{P}(\bar{V} \leqslant y)+2 \varepsilon+\varepsilon \Delta^{-1} \bar{\sigma} \sqrt{2 \pi}
$$

а нижний предел не меньше, чем

$$
\mathbf{P}(\bar{V} \leqslant y)-\varepsilon \bar{\sigma} \sqrt{2 \pi} \Delta^{-1}-3 \varepsilon-\varepsilon^{2},
$$

что и завершает доказательство леммы. 
Положим при любых $A \subseteq(-\infty, \infty), B \subseteq[1, \infty))$

$$
\begin{aligned}
\hat{I}_{n}(A \times B) & =\int_{A} \int_{B} v^{-1} \exp \left\{-\bar{h} u-v^{-1} e^{-u}\right\} \mu_{n}(d u, d v), \\
I(A \times B) & =\int_{A} \int_{B} v^{-1} \exp \left\{-\bar{h} u-v^{-1} e^{-u}\right\} d u d G_{\theta}(v),
\end{aligned}
$$

где

$$
G_{\theta}(v)=\mathbf{P}(\bar{V} \leqslant v), \quad \mu_{n}(d u, d v)=\mathbf{P}\left(\hat{S}_{n} \in d u, \bar{V}_{n} \in d v\right)
$$

(см. (46), (23)).

Лемма 6. Для любых $A \subseteq[-\beta n, \infty), \theta / 2<\beta<\theta, B \subseteq[1, \infty)$ nри $n \rightarrow \infty$ имеет место соотношение (см. (31))

$$
I_{n}(A \times B) \sim \hat{I}_{n}(A \times B) .
$$

Доказательство. Проведем оценки функции $g_{n}(u, v)$ (см. (30)) снизу и сверху. Подставив $x=v^{-1} e^{-\theta n-u}$ в неравенство

$$
x(1-x / 2) \leqslant \ln (1+x) \leqslant x, \quad x \geqslant 0 .
$$

и, учитывая, что $v \geqslant 1$, получаем, что

$$
v^{-1} e^{-\theta n-u}\left(1-(1 / 2) e^{-\theta n-u}\right) \leqslant \ln \left(1+v^{-1} e^{-\theta n-u}\right) \leqslant v^{-1} e^{-\theta n-u}
$$

Домножив неравенства (70) на $-k_{n}\left(k_{n}\right.$ - целая часть числа $\left.e^{\theta n}\right)$ и потенцируя, получаем оценки

$$
\begin{aligned}
\exp \left\{-v^{-1} e^{-u} k_{n} e^{-\theta n}\right\} & \leqslant\left(1+v^{-1} e^{-\theta n-u}\right)^{-k_{n}} \\
& \leqslant \exp \left\{-v^{-1} e^{-u} k_{n} e^{-\theta n}\left(1-(1 / 2) e^{-\theta n-u}\right)\right\}
\end{aligned}
$$

Из неравенств (71) выводим, что при $n \rightarrow \infty$

$$
\left(1+v^{-1} e^{-\theta n-u}\right)^{-k_{n}} \sim \exp \left\{-v^{-1} e^{-u}\right\}
$$

равномерно по $v \geqslant 1$ и $u$ таким, что при $n \rightarrow \infty$

$$
\theta n+2 u \rightarrow \infty
$$

Для $u \geqslant-\beta n$ при $\beta>\theta / 2$ условие (73) выполнено, и кроме того, при $n \rightarrow \infty$ равномерно по этим $u$ и по $v \geqslant 1$

$$
\left(v+e^{-\theta n-u}\right)^{-1} \sim v^{-1}
$$

Перемножая почленно соотношения (72) и (74), получаем, что при $n \rightarrow \infty$ равномерно по $u \geqslant-\beta n, v \geqslant 1$

$$
g_{n}(u, v) \sim \exp \left\{-v^{-1} e^{-u}\right\} .
$$

Подставив соотношение (75) в (31), приходим к (69). 
Лемма 7. Интеграл $I(\theta)$, определенный соотночением (22), конечен и имеет место равенство (см. (68))

$$
I(\theta)=I((-\infty, \infty) \times[1, \infty)) .
$$

Доказательство. Проведем замену переменного $x=v^{-1} e^{u}$ и получим равенство

$$
\int_{-\infty}^{\infty} \exp \left\{-\bar{h} u-v^{-1} e^{-u}\right\} d u=v^{\bar{h}} \Gamma(\bar{h})
$$

Интеграл

$$
\int_{1}^{\infty} v^{\bar{h}-1} d G_{\theta}(v)
$$

очевидно, конечен при $\bar{h} \leqslant 1$ (и не превосходит 1). При $\bar{h}>1$, воспользовавшись формулой для моментов неотрицательной случайной величины $\xi$

$$
\mathbf{E} \xi^{\varkappa}=\int_{0}^{\infty} \varkappa x^{\varkappa-1} \mathbf{P}(\xi>x) d x, \quad \varkappa>0,
$$

и вторым из неравенств (44), получим для интеграла (77) оценку сверху

$$
\bar{C}(\bar{h}-1) \int_{1}^{\infty} v^{-2} d v=\bar{C}(\bar{h}-1) .
$$

Таким образом, повторный интеграл

$$
\int_{1}^{\infty} v^{-1} d G_{\theta}(v) \int_{-\infty}^{\infty} \exp \left\{-\bar{h} u-v^{-1} e^{-u}\right\} d u=\Gamma(\bar{h}) \int_{1}^{\infty} v^{\bar{h}-1} d G_{\theta}(v)
$$

конечен и верно равенство

$$
I(\theta)=I((-\infty, \infty) \times[1, \infty)) .
$$

Лемма 8. Для любых $a>0, b>1$ при $n \rightarrow \infty$ имеет место соотношение

$$
\bar{\sigma} \sqrt{2 \pi n} \hat{I}_{n}([-a, a] \times[1, b]) \rightarrow I([-a, a] \times[1, b]),
$$

Доказательство. Введем на $[-a, a] \times[1, b]$ последовательность мер

$$
v_{n}(d u, d v)=\bar{\sigma} \sqrt{2 \pi n} \mu_{n}(d u, d v) .
$$

Из леммы 5 следует, что последовательность $v_{n}$ слабо сходится к мере $d u \times d G_{\theta}(v)$. Поскольку подынтегральная функция в $\hat{I}_{n}$ непрерывна, отсюда вытекает (78).

Лемма 9. Для любых $a, \varepsilon>0$ найдется такое $b>1$, что

$$
\hat{I}_{n}([-a, a] \times[b, \infty))<\varepsilon / \sqrt{n} .
$$

Доказательство. Проводя элементарные выкладки и применяя первое из неравенств (44), получаем, что

$$
\begin{aligned}
& \int_{-a}^{a} \int_{b}^{\infty} v^{-1} \exp \left\{-\bar{h} u-v^{-1} e^{-u}\right\} \mu_{n}(d u, d v) \leqslant b^{-1} e^{\bar{h} a} \mathbf{P}\left(\hat{S}_{n} \in[-a, a], \bar{V}_{n} \geqslant b\right) \\
& \leqslant 2 a \bar{C} b^{-1-\bar{h}} e^{\bar{h} a} n^{-1 / 2} \text {. }
\end{aligned}
$$

Остается при заданном $a$ выбрать $b$ из условия

$$
2 \bar{C} b^{-1-\bar{h}} a e^{\bar{h} a}<\varepsilon .
$$


Лемма 10. Для любого $\varepsilon>0$ найдется такое $a>0$, что

$$
\hat{I}_{n}((a, \infty) \times[1, \infty))<\varepsilon / \sqrt{n} .
$$

Доказательство. Очевидно, что (см. (67))

$$
\hat{I}_{n}((a, \infty) \times[1, \infty)) \leqslant \int_{a+}^{\infty} e^{-\bar{h} u} d \mathbf{P}\left(\hat{S}_{n} \leqslant u\right)
$$

Таким образом, следует установить, что при подходящем $a$

$$
\int_{a+}^{\infty} e^{-\bar{h} u} d \mathbf{P}\left(\hat{S}_{n} \leqslant u\right) \leqslant \varepsilon / \sqrt{n} .
$$

Неравенство (82) легко выводится из доказательства теоремы Петрова [8]. Именно, проинтегрируем (82) по частям, заметим, что внеинтегральный член при этом равен нулю, воспользуемся неравенством Берри-Эссеена (см. (13)) и, снова применяя интегрирование по частям, получим, что

$$
\begin{aligned}
& \left.\mathbf{P}\left(a<\hat{S}_{n} \leqslant u\right) e^{-\bar{h} u}\right|_{a} ^{\infty}+\int_{a}^{\infty} \mathbf{P}\left(a<\hat{S}_{n} \leqslant u\right) \bar{h} e^{-\bar{h} u} d u \\
& \leqslant \int_{a}^{\infty}\left(\Phi\left(\frac{u}{\bar{\sigma} \sqrt{n}}\right)-\Phi\left(\frac{a}{\bar{\sigma} \sqrt{n}}\right)\right) \bar{h} e^{-\bar{h} u} d u+\frac{2 \mathscr{A} \bar{\rho}^{*}}{\sqrt{n}} \int_{a}^{\infty} \bar{h} e^{-\bar{h} u} d u \\
& =\int_{a}^{\infty} e^{-\bar{h} u} d \Phi(u /(\bar{\sigma} \sqrt{n}))+\left(2 A \bar{\rho}^{*} / \sqrt{n}\right) e^{-\bar{h} a} .
\end{aligned}
$$

Интеграл в (83) равен

$$
\frac{1}{\bar{\sigma} \sqrt{2 \pi n}} \int_{a}^{\infty} e^{-\bar{h} u} e^{-u^{2} /\left(\bar{\sigma}^{2} n\right)} d u \leqslant \frac{1}{\bar{h} \bar{\sigma} \sqrt{2 \pi n}} e^{-\bar{h} a} .
$$

Подставляя (84) в (83), приходим к (82).

Лемма 11. Для любых $\varepsilon, \beta>0$ найдется такое $a>0$, что

$$
\hat{I}_{n}((-\beta n,-a] \times[1, \infty))<\varepsilon / \sqrt{n} .
$$

Доказательство. Для упрощения записи заменим в интеграле (85) переменную $u$ на $-u$, а переменную $v$ на $w^{-1}$ и приведем его к виду

$$
\int_{a}^{\beta n} \int_{0}^{1} w \exp \left\{\bar{h} u-w e^{u}\right\} \mathbf{P}\left(-\hat{S}_{n} \in d u, \bar{V}_{n}^{-1} \in d w\right) .
$$

Функщия $y=w \exp \left\{-w e^{u}\right\}$ переменного $w$ (при $u>0$ ) возрастает при $w \in\left[0, e^{-u}\right]$ и убывает при $w \in\left[e^{-u}, 1\right]$, достигая при $w=e^{-u}$ максимального значения $e^{-u-1}$. Пусть $2 \bar{h} a>1$; разобьем область внутреннего интегрирования в $(86)$ на части $\left(0,2 \bar{h} u e^{-u}\right]$ и $\left(2 \bar{h} u e^{-u}, 1\right]$. Будем считать $a$ настолько большим, что $2 \bar{h} a e^{-a}<1$, и представим интеграл (86) в виде

$$
\int_{a}^{\beta n}\left(\int_{0}^{2 \bar{h} u e^{-u}}+\int_{2 \bar{h} u e^{-u}}^{1}\right) w \exp \left\{\bar{h} u-w e^{u}\right\} \mathbf{P}\left(-\hat{S}_{n} \in d u, \bar{V}_{n}^{-1} \in d w\right) .
$$


При фиксированном $u$ и $w \in\left(0,2 \bar{h} u e^{-u}\right]$ оценим подынтегральную функцию в (87) ее максимальным значением $\exp \{\bar{h} u-u-1\}$. В результате соответствующий двойной интеграл заменяется на простой интеграл

$$
\int_{a}^{\beta n} \exp \{(\bar{h}-1) u-1\} \mathbf{P}\left(-\hat{S}_{n} \in d u, \bar{V}_{n} \geqslant(2 \bar{h} u)^{-1} e^{u}\right) .
$$

При $w \in\left(2 \bar{h} u e^{-u}, 1\right]$ подынтегральную функцию в (87) также заменяем на ее максимум - значение в левом конце промежутка интегрирования:

$$
2 \bar{h} u e^{-u} \exp \left\{\bar{h} u-2 \bar{h} u e^{-u} e^{u}\right\}=2 \bar{h} u \exp \{-\bar{h} u-u\} .
$$

Во втором двойном интеграле в (87) при интегрировании отбросим событие $\bar{V}_{n}^{-1} \epsilon$ $\left(2 \bar{h} u e^{-u}, 1\right]$ и получим границу сверху в виде

$$
2 \bar{h} \int_{a}^{\beta n} u \exp \{-(\bar{h}+1) u\} \mathbf{P}\left(-\hat{S}_{n} \in d u\right) \leqslant \varepsilon / \sqrt{n},
$$

где неравенство (89) устанавливается так же, как (82).

Для интеграла $(88)$ при $\bar{h}<1$ получаем оценку

$$
e^{-1} \int_{a}^{\beta n} \exp \{-(1-\bar{h}) u\} \mathbf{P}\left(-\hat{S}_{n} \in d u\right) \leqslant \varepsilon / \sqrt{n}
$$

по тем же соображениям, что и в (82).

При $\bar{h} \geqslant 1$ введем разбиение

$$
\Delta_{i}=(a+i \delta, a+(i+1) \delta), \quad i=0,1,2, \ldots,
$$

при произвольном $\delta>0$ и, полагая для простоты $\beta n$ кратным $\delta$, заменим интеграл (88) суммой интегралов

$$
\begin{aligned}
& e^{-1} \sum_{i} \int_{\Delta_{i}} \exp \{(\bar{h}-1) u\} \mathbf{P}\left(-\hat{S}_{n} \in d u, \bar{V}_{n} \geqslant(2 \bar{h} u)^{-1} e^{u}\right) \\
& \quad \leqslant e^{-1} \sum_{i}\left(\exp \{(\bar{h}-1)(a+(i+1) \delta)\} \mathbf{P}\left(-\hat{S}_{n} \in \Delta_{i}, \bar{V}_{n}>(2 \bar{h}(a+i \delta))^{-1} e^{a+i \delta}\right)\right) .
\end{aligned}
$$

Применяя лемму 4, оценим вероятности под знаком суммы (91) сверху выражением

$$
\frac{C \delta \bar{C}}{\sqrt{n}}\left(\left(1-e^{-\bar{\alpha}}\right)(2 \bar{h}(a+i \delta))^{-1} e^{a+i \delta}\right)^{-\bar{h}}=\frac{C_{\bar{h}, \bar{\sigma}} \delta}{\sqrt{n}}(a+i \delta)^{\bar{h}} e^{-\bar{h}(a+i \delta)} .
$$

Подставим (92) в (91) и получим неравенство

$$
\frac{C_{\bar{h}, \bar{\sigma}}}{\sqrt{n}} e^{(\bar{h}-1) \delta-1} \sum_{i}(a+i \delta)^{\bar{h}} e^{-(a+i \delta)} \delta \leqslant \frac{C_{\bar{h}, \bar{\sigma}}}{\sqrt{n}} e^{(\bar{h}-1) \delta-1} \int_{a}^{\beta n} u^{\bar{h}} e^{-u} d u .
$$

Собирая вместе оценки (89), (90), (92), приходим к заключению леммы.

Леммы 10, 11, 6, 7 приводят к соотношению (32), откуда, как было отмечено ранее, следует асимптотика (33) для математического ожидания $\mathbf{E}_{1}^{(n)}$. Перейдем к оценке $\mathbf{E}_{2}^{(n)}$. 
Лемма 12. Для любого $0<\beta<\theta$ при $n \rightarrow \infty$ имеет место соотношение

$$
\mathbf{E}\left(V_{n+1}^{-1}\left(1+V_{n}^{-1} U_{n}\right)^{-k_{n}} ; S_{n}<\beta n\right)=o(1) \mathbf{P}\left(S_{n}>\theta n\right),
$$

Доказательство. Положим

$$
m_{n}=\min _{0 \leqslant j \leqslant n} S_{j}
$$

Заметив, что

$$
e^{-m_{n}} \leqslant V_{n+1} \equiv \sum_{j=0}^{n} e^{-S_{j}}, \quad V_{n} \leqslant V_{n+1},
$$

запишем для математического ожидания (94) оценку сверху в виде

$$
\mathbf{E}\left(e^{m_{n}}\left(1+(n+1)^{-1} e^{m_{n}-S_{n}}\right)^{-k_{n}} ; S_{n}<\beta n\right)=E_{21}^{(n)}+E_{22}^{(n)}+E_{23}^{(n)},
$$

где $E_{21}^{(n)}, E_{22}^{(n)}$ и $E_{23}^{(n)}$ обозначают математические ожидания, взятые по пересечению события $S_{n}<\beta n$, соответственно, с событиями

$$
\left\{S_{n}-m_{n}>(\theta+\varepsilon) n\right\}, \quad\left\{\left|S_{n}-m_{n}-\theta n\right| \leqslant \varepsilon n\right\}, \quad\left\{S_{n}-m_{n}<(\theta-\varepsilon) n\right\} .
$$

Очевидно, что

$$
\begin{aligned}
E_{21}^{(n)} & \leqslant \mathbf{P}\left(S_{n}-m_{n}>(\theta+\varepsilon) n\right) \\
& =\sum_{k=1}^{n-1} \mathbf{P}\left(S_{n}-S_{k}>(\theta+\varepsilon) n, S_{j}>S_{k}, j<k, S_{j} \geqslant S_{k}, k \leqslant j \leqslant n-1\right) .
\end{aligned}
$$

Пусть $\varepsilon>0$ настолько мало, что $\theta+\varepsilon<\theta^{+}$. Применяя (12), получим, что при $1 \leqslant k \leqslant n-1$

$$
\mathbf{P}\left(S_{n}-S_{k}>(\theta+\varepsilon) n\right) \leqslant e^{-h(\theta+\varepsilon)(\theta+\varepsilon) n} R(h(\theta+\varepsilon))^{n-k} \leqslant e^{-\Lambda(\theta+\varepsilon) n} .
$$

Подставим (98) в (97) и получим оценку

$$
E_{21}^{(n)} \leqslant n e^{-\Lambda(\theta+\varepsilon) n},
$$

которая, очевидно, удовлетворяет требованию (94). Далее,

$$
\begin{aligned}
E_{22}^{(n)} & \leqslant \mathbf{E}\left(e^{m_{n}} ;\left|S_{n}-m_{n}-\theta n\right| \leqslant \varepsilon n, S_{n}<\beta n\right) \\
& \leqslant e^{(\beta-\theta+\varepsilon) n} \mathbf{P}\left(S_{n}-m_{n}>(\theta-\varepsilon) n\right) \leqslant e^{(\beta-\theta+\varepsilon) n} n e^{-\Lambda(\theta-\varepsilon) n},
\end{aligned}
$$

где последнее неравенство выводится аналогично (99). Пусть $\varepsilon$ выбрано настолько малым, что $\theta-\beta-\varepsilon+\Lambda(\theta-\varepsilon)>\Lambda(\theta)$. В таком случае неравенство (100) дает оценку требуемого типа (94). Наконец, при $S_{n}-m_{n}<(\theta-\varepsilon) n$

$$
\left(1+(n+1)^{-1} e^{-\left(S_{n}-m_{n}\right)}\right)^{-k_{n}} \leqslant\left(1+(n+1)^{-1} e^{-(\theta-\varepsilon) n}\right)^{-e^{\theta n}+1} .
$$

Подставляя неравенство (101) в математическое ожидание $E_{23}^{(n)}$, получаем и для него требуемую оценку типа (94). Тем самым лемма доказана, а вместе с ней закончено доказательство теоремы 1. 


\section{5. Доказательство теоремы 2}

Предположение $\mu \geqslant 0$ в доказательстве теоремы 1 впервые использовалось в той части леммы 2, где утверждалось, что в неравенстве (35) при достаточно малых $\alpha$ можно положить $q_{\alpha}=\bar{h}$. Отсюда выводились неравенства (44), которые использовались для доказательства лемм 7,11 . Остальные леммы либо не опираются на предположение $\mu \geqslant 0$, либо там, где используются неравенства (44), годятся неравенства (43). Внесем необходимые дополнения в доказательство лемм 7,11 при $\mu<0$. Если $\bar{h}$ таково, что $R_{X}(\bar{h}) \geqslant 1$, то лемма 2 полностью сохраняется, и при $\alpha \leqslant \bar{\alpha}$ можно положить в (12) $q_{\alpha}=\bar{h}$, и следовательно, верны неравенства (44), и последующее доказательство теоремы 1 переносится без изменений.

Предположим далее, что $R_{X}(\bar{h})<1$. Функция $R_{X}(h)$ строго выпукла (вниз) и непрерывна, так что существует единственное $\bar{q} \in(0, h)$, для которого выполняется равенство

$$
R_{X}(\bar{h}-\bar{q})=R_{X}(\bar{h}) .
$$

Поскольку $\bar{h}=h(\theta)>h(0)$ при $\theta>0$ и $R_{X}^{\prime}(h(0))=0$, справедливо неравенство $\cdot \bar{h}-\bar{q}<h(0)$. Таким образом,

$$
R_{-\bar{X}}(\bar{q})=R_{X}(\bar{h})^{-1} R_{X}(\bar{h}-\bar{q})=1 .
$$

Так как $\mathbf{E}(-\bar{X})=-m_{X}(\bar{h})<0$, применяя лемму 1 , заключаем, что

$$
\mathbf{P}\left(\max _{0 \leqslant k \leqslant n}\left(-\bar{S}_{k}\right)>y\right) \leqslant e^{-\bar{q} y} .
$$

Отсюда следует, что показатель $q_{\alpha}$ экспоненты в неравенствах (35), (43) стремится к $\bar{q}$ при $\alpha \rightarrow 0, \alpha>0$.

Для доказательства соотношения (76) леммы 7 надо в условиях теоремы 2 установить конечность интеграла (77). Из доказательства леммы 7 следует оценка сверху

$$
\left(1-e^{-\alpha}\right)^{-q_{\alpha}}(\bar{h}-1) \int_{1}^{\infty} v^{\bar{h}-q_{\alpha}-2} d v
$$

(где в условиях теоремы 1 можно было положить $q_{\alpha}=\bar{h}$ ). Интеграл в (103) сходится, если $\bar{h}-q_{\alpha}<1$. Если $\bar{h}-\bar{q}<1$, то, выбирая $\alpha>0$ достаточно малым, получим требуемый результат. Неравенство $\bar{h}-\bar{q}<1$ следует из неравенства $\bar{h}-\bar{q}<h(0)$ при $h(0) \leqslant 1$, а при $h(0)>1, \bar{h}>h^{*}$ следует из определения величины $h^{*}$.

В лемме 11 модификации требует только оценка (93). Просматривая предшествующие ей рассуждения, получаем в рассматриваемом случае, что оценка (92) с точностью до постоянного множителя равна

$$
\delta n^{-1 / 2}(a+i \delta)^{q_{\alpha}} e^{-q_{\alpha}(a+i \delta)} .
$$

В результате приходим к следующей модификации оценки (93) без учета постоянного множителя:

$$
n^{-1 / 2} e^{(\bar{h}-1) \delta} \sum_{i}(a+i \delta)^{q_{\alpha}} e^{\left(\bar{h}-q_{\alpha}-1\right)(a+i \delta)} \delta \leqslant n^{-1 / 2} e^{(\bar{h}-1) \delta} \int_{a}^{\beta n} u^{q_{\alpha}} e^{\left(\bar{h}-q_{\alpha}-1\right) u} d u .
$$

Остается заметить, что, как было указано выше, $\bar{h}-q_{\alpha}-1<0$ при достаточно малом $\alpha>0$ и интеграл в (104) может быть сделан как угодно малым за счет выбора $a$, что и требуется в лемме 11 . Теорема 2 доказана. 


\section{6. Заключительные замечания}

В соответствии с классификацией при $\mu>0, \mu=0$ и $\mu<0$ ветвящийся процесс в случайной среде называется, соответственно, надкритическим, критическим и докритическим; последние разделяются на умеренно $\left(R^{\prime}(1)>0\right)$, промежуточно $\left(R^{\prime}(1)=0\right)$ и строго $\left(R^{\prime}(1)<0\right)$ докритические. Теорема 1 полностью описывает асимптотику вероятностей больших уклонений в надкритическом и критическом случаях, а теорема 2 для умеренно и промежуточно докритических процессов. В случае строго докритического процесса теорема 2 описывает область больших уклонений, где асимптотика вероятностей $\mathbf{P}\left(\ln Z_{n}>\theta n\right)$ следует за асимптотикой вероятностей $\mathbf{P}\left(S_{n}>\theta n\right)$. Для строго докритического процесса при $\theta \leqslant \theta^{*}$ асимптотика вероятностей $\mathbf{P}\left(\ln Z_{n}>\theta n\right)$ и $\mathbf{P}\left(S_{n}>\theta n\right)$ различна. Случай, когда $R^{\prime}(h)<0$ при всех $h \in\left(\mu, h^{+}\right)$, в работе не рассматривался, так как при этом асимптотика (9) для $\theta>0$ не выполняется.

Результаты аналогичные теоремам 1, 2 верны для производящих функций общего вида (2), мы вернемся к этому в другом месте.

Пользуюсь возможностью выразить благодарность анонимному рецензенту, исключительно внимательно прочитавшему работу и указавшему на большое число опечаток и неточностей.

\section{Список литературы}

1. Agresti A., On the extinction times of varying and random environment branching processes. J. Appl. Prob. (1975) 12, №1, 39-46.

2. Афанасьев В. И., О соотношении максимального и общего числа частиц в критическом ветвящемся процессе в случайной среде. Теория вероятностей и ее применения (2003) 48, №3, $435-452$.

3. Ватутин В. А., Предельная теорема для промежуточно докритического ветвящегося процесса в случайной среде. Теория вероятностей и ее применения (2003) 48, №3, 453-465.

4. Альбеверио С., Козлов М. В., О возвратности и транзиентности зависящих от состояния ветвящихся процессов в случайной среде. Теория вероятностей и ее применения (2003) 48, №4, $641-660$.

5. Geiger I., Kersting G, The survival probability of a critical branching process in random environment. Теория вероятностей и ее применения (2000) 45, №3, 607-615.

6. Козлов М. В., Об асимптотике вероятности невырождения критических ветвящихся процессов в случайной среде. Теория вероятностей и ее применения (1976) 21, №4, 813-825.

7. Козлов М. В., Условная функциональная предельная теорема для критического ветвящегося процесса в случайной среде. Докл. РАН (1995) 344, №1, 12-15.

8. Петров В. В. О вероятности больших уклонений сумм независимых случайных величин. Теория вероятностей и ее применения (1965) 10, №2, 310-322.

9. Петров В. В., Суммы независимых случайных величин. Наука, Москва, 1972.

10. Феллер В., Введение в теорию вероятностей и ее приложения, т. 2. Москва, Мир, 1984.

11. Боровков А. А., Вероятностные прочессы в теории массового обслуживания. Наука, Москва, 1972. 